\title{
Um Framework de Práticas Ágeis para Apoio à Implementação do Processo de Projeto e Construção do Produto do MR-MPS-SW
}

\author{
Vitor de Souza Castro ${ }^{1}$, Sandro Ronaldo Bezerra Oliveira ${ }^{1}$, Alexandre Marcos \\ Lins de Vasconcelos ${ }^{2}$
}

${ }^{1}$ Programa de Pós-Graduação em Ciência da Computação - Instituto de Ciências Exatas e Naturais - Universidade Federal do Pará (UFPA), Caixa Postal 15.064 - 91.501-970 Belém - PA - Brasil

${ }^{2}$ Centro de Informática - Universidade Federal de Pernambuco (UFPE) Caixa Postal 7851 - 50.740-560 - Recife - PE - Brasil

castro.vitor006@gmail.com, srbo@ufpa.br, amlv@cin.ufpe.br

\begin{abstract}
Software quality is a recurring theme in software development companies. In Brazil, the SOFTEX created the MPS.BR program, with aims to boost the quality of Brazilian software. Additionally, seeking greater agility in processes, many companies are adopting agile practices to support the implementation of these quality program, specifically the reference model for software, the MR-MPS-SW. Thus, this paper presents a framework containing agile practices derived of Crystal, FDD, TDD and eXtreme Programming methodologies to support the implementation of the Product Design and Construction process included in MPS.BR program. This framework was evaluated from peer review with expert.
\end{abstract}

Resumo. Qualidade de software é um tema recorrente nas organizações especializadas. No Brasil, a SOFTEX criou o programa MPS.BR, tendo o objetivo de impulsionar a qualidade do software brasileiro. Adicionalmente, visando maior agilidade nos processos, muitas organizações estão adotando práticas ágeis para apoiar a implementação deste programa de qualidade, especificamente o modelo referência para software, o MR-MPS-SW. Assim, o presente artigo visa apresentar um framework contendo práticas ágeis oriundas das metodologias Crystal, FDD, TDD e eXtreme Programming para apoio à implementação do processo de Projeto e Construção do Produto constante no programa MPS.BR. Este framework foi avaliado a partir de uma revisão por pares com especialista.

\section{Introdução}

Organizações buscam com o apoio da tecnologia da informação diferenciais competitivos frente aos concorrentes. Para a construção de sistemas computacionais, softwares, aplicativos e outros, atividades de especificação, projeto, implementação, validação e evolução são fundamentais para o processo de desenvolvimento de software [Sommervile, 2007]. 
No contexto relacionado à atividade de construção da solução, existem inúmeros procedimentos, técnicas e metodologias para apoiar o desenvolvimento dessa atividade, visando a qualidade e a padronização do processo. Neste contexto, a Engenharia de Software é uma abordagem sistemática e disciplinada para o desenvolvimento de software [Pressman, 2011].

Visando a diferenciação no mercado, organizações estão buscando a implementação de programas de qualidade, pois apresentam objetivos específicos a serem atingidos com o enfoque na qualidade do software, tendo como exemplo o modelo CMMI (Capability Maturity Model Integration) [SEI, 2010] e o MPS.BR (Melhoria do Processo de Software Brasileiro) [SOFTEX, 2013].

Por outro lado, influenciados pelo Toyotismo, cujo principio está baseado na produção "enxuta" [Poppendieck, 2011], as metodologias ágeis apresentam-se como uma forma menos burocrática para a aplicação da Engenharia de Software. No cenário em que organizações estão em busca de processos "leves" e com qualidade [Sbrocco, 2012], torna-se cada vez mais presente a aplicação dos modelos de qualidade com o apoio das metodologias ágeis.

Assim, esta pesquisa tem o objetivo de apresentar um framework de práticas ágeis para apoio aos modelos de qualidade CMMI e MPS.BR, especificamente para a área de processo de Solução Técnica, constante no CMMI, e o processo de Projeto e Construção do Produto (PCP), constante no MPS.BR. No contexto da aplicação do modelo MPS.BR, até Julho/2015, apenas 55 empresas já tiveram seus processos implementados nos resultados esperados do nível D, nível em que o processo PCP está inserido. Além disso, 594 empresas, totalizando $92 \%$ do total de empresas avaliadas, estão em níveis inferiores de maturidade [SOFTEX, 2015]. Apesar do elevado índice de empresas avaliadas em níveis inferiores de maturidade, o percentual restante são de empresas que estão nos níveis A, B, C e D, e precisam manter as práticas do nível D alinhadas aos objetivos do programa MPS.BR para preservar a avaliação. Isso pode denotar que, nos próximos anos, as organizações que continuarem com o programa do MPS.BR estarão atingindo ou mantendo o nível D e com isso há necessidade de fornecer um framework de apoio para a implementação desse processo.

As metodologias ágeis utilizadas para compor o framework foram: Crystal [Cockburn, 2002], eXtreme Programming - XP [Beck, 2004], Feature Driven Development - FDD [Retamal, 2008] e Test-Driven Development - TDD [Beck, 2003]. A seleção das metodologias para compor o framework esteve pautada nas práticas ágeis e no apoio dessas práticas ao processo de PCP.

Assim, pode-se declarar como partes desta pesquisa:

- Questão de Pesquisa: identificar como os métodos ágeis podem apoiar a implementação de processos constantes em modelos de qualidade;

- Hipótese: a partir de um framework (arcabouço) de práticas ágeis consultores recebem um apoio para a implementação mais gradativa de processos constantes em modelos de qualidade;

- Suposição: a partir de um instrumento de apoio para a implementação de processos constantes em modelos de qualidade, consultores e empresas

CASTRO, V. S.; OLIVEIRA, S. R. B.; VASCONCELOS, A. M. L.

Um Framework de Práticas Ágeis para Apoio à Implementação do Processo de Projeto e Construção do Produto do MR-MPS-SW

isys - Revista Brasileira de Sistemas de Informação, Rio de Janeiro, vol. 8, No. 2, p. 78-97 2015 
interessadas nestes modelos podem ganhar produtividade e assertividade na implementação dos resultados esperados presentes nestes processos.

Além dessa seção introdutória, este artigo está estruturado em mais cinco outras seções: a seção 2 apresenta os trabalhos relacionados; a seção 3 apresenta o processo de Projeto e Construção do Produto e seus resultados esperados; a seção 4 detalha o framework, a metodologia utilizada, os objetivos e as práticas; a seção 5 contempla o processo da revisão por pares, utilizado para avaliação do framework; por fim, a seção 6 apresenta as considerações finais do trabalho, principais limitações e alguns trabalhos futuros.

\section{Trabalhos Relacionados}

$\mathrm{Na}$ atividade de revisão da literatura foram identificados diversos trabalhos, como pode ser melhor detalhado em [Castro et al., 2015], dentre os quais pode-se destacar o trabalho de Lukasiewicz et al. (2010), que apresenta o mapeamento do Scrum a 123 objetivos específicos do CMMI. Pelo não atendimento a todos os objetivos específicos do CMMI, novas práticas foram incluídas para manter a agilidade. A problemática da aplicação dos métodos ágeis com modelos de qualidade é destacada. No mapeamento realizado há 8 práticas do CMMI atendidas, sendo 2 com atendimento Total, 3 Parcial e 3 Inexistentes para a área de processo de Solução Técnica. Além disso, nesse trabalho não há o detalhamento e a forma de implementação das práticas ágeis.

O trabalho de Catunda et al. (2011) está centrado na implantação do MPS.BR em uma organização. A abordagem das práticas é muito ampla no sentido que não houve a associação direta das práticas aos resultados esperados. Na etapa de análise, projeto e construção do produto não são evidenciadas práticas ou metodologias de apoio para a implementação.

Já no trabalho de Silva et al. (2009), a análise de aderência aos resultados esperados em todos os níveis do modelo MPS.BR são realizadas para as metodologias FDD e Scrum. A limitação do trabalho está na análise de somente duas metodologias e na perspectiva da especificação de quais práticas são necessárias para atingir cada resultado esperado. Também não há justificativas que evidenciem o atendimento das metodologias para cada nível do MPS.BR.

Por fim, o trabalho de Hansen et al. (2009) apresenta um relato de experiência no uso de abordagens ágeis para o desenvolvimento de software. Inicialmente foi realizada a implantação do CMMI 3 e depois as abordagens ágeis foram utilizadas. Investimentos em treinamento para a institucionalização do Scrum na organização foram realizados. Além disso, nesse artigo não há o detalhamento da implementação das abordagem ágeis, e além disso a associação da prática ágil ao processo não é realizada.

Assim, com esta revisão fica claramente nítida a necessidade de pesquisas e experimentações na academia e na indústria de software sobre o uso integrado de métodos ágeis e modelos de qualidade para o alcance dos objetivos dos processos de Engenharia de Software, uma vez que dentre todos os trabalhos relatados sobre implementação de programas de qualidade, cerca de $60 \%$ tratavam da associação entre estes temas de pesquisa. Isso pode ajudar a comunidade a desmistificar o seguinte pensamento: o desenvolvimento ágil busca tratar o processo de produção de software de maneira

CASTRO, V. S.; OLIVEIRA, S. R. B.; VASCONCELOS, A. M. L.

Um Framework de Práticas Ágeis para Apoio à Implementação do Processo de Projeto e Construção do Produto do MR-MPS-SW

iSys - Revista Brasileira de Sistemas de Informação, Rio de Janeiro, vol. 8, No. 2, p. $78-97$ 2015 
diferenciada, uma vez que entende que se trata de um processo criativo e que produz um bem intangível; já os modelos de qualidade baseiam-se no pensar fordista, tratando a produção de software de maneira parecida com os produtos manufaturados.

\section{O Processo de Projeto e Construção do Produto}

A SOFTEX - Associação para a Promoção do Software Brasileiro, desenvolveu o programa MPS.BR - Melhoria do Processo de Software Brasileiro [SOFTEX, 2013], com o objetivo de impulsionar a melhoria da maturidade e da capacidade do desenvolvimento de software e serviços nas empresas brasileiras. No contex to desse programa, o MR-MPSSW - Modelo de Referência do MPS para Software, tem como base os requisitos de processos definidos nos modelos de melhoria de processo e atende a necessidade de implantar os princípios de engenharia de software de forma adequada [SOFTEX, 2013].

No MR-MPS-SW há 7 níveis de maturidades, que vão do $\mathrm{G}$ até o $\mathrm{A}$. O processo de Projeto e Construção do Produto está situado no nível D. O propósito deste processo é projetar, desenvolver e implementar soluções para atender aos requisitos [SOFTEX, 2013]. Para atendimento ao propósito definido, foram estabelecidos 8 resultados esperados (vide Tabela 1). Segundo a ISO/IEC (2004), resultado esperado é um resultado observável do sucesso do alcance do propósito do processo. Além disso, a Tabela 1 contém os objetivos principais para o atendimento de cada resultado esperado.

\section{Tabela 1. Resultados Esperados do processo PCP do MR-MPS- SW}

\begin{tabular}{|l|l|}
\multicolumn{1}{|c|}{ Resultado Esperado } & \multicolumn{1}{|c|}{ Objetivo } \\
\hline $\begin{array}{l}\text { PCP1. Alternativas de solução e critérios de seleção são } \\
\text { desenvolvidos para atender aos requisitos definidos de } \\
\text { produto e componentes de produto. }\end{array}$ & $\begin{array}{l}\text { Identificar possíveis soluções para a } \\
\text { construção do produto baseado em critérios } \\
\text { pré-estabelecidos. }\end{array}$ \\
\hline $\begin{array}{l}\text { PCP2. Soluções são selecionadas para o produto ou } \\
\text { componentes do produto, com base em cenários definidos } \\
\text { e em critérios identificados }\end{array}$ & $\begin{array}{l}\text { Selecionar as soluções alternativas e } \\
\text { reavaliá-las }\end{array}$ \\
\hline $\begin{array}{l}\text { PCP3. O produto e/ou componente do produto é projetado } \\
\text { e documentado }\end{array}$ & Realizar e documentar o design do produto. \\
\hline $\begin{array}{l}\text { PCP4. As interfaces entre os componentes do produto são } \\
\text { projetadas com base em critérios predefinidos }\end{array}$ & Projetar as interfaces (serviços) do produto \\
\hline $\begin{array}{l}\text { PCP5. Uma análise dos componentes do produto é } \\
\text { conduzida para decidir sobre sua construção, compra ou } \\
\text { reutilização }\end{array}$ & $\begin{array}{l}\text { Definir como será concebido o produto, } \\
\text { podendo ser por desenvolvimento interno, } \\
\text { aquisição ou reutilização. }\end{array}$ \\
\hline $\begin{array}{l}\text { PCP6. Os componentes do produto são implementados e } \\
\text { verificados de acordo com o que foi projetado } \\
\text { disponibilizada de acordo com os padrões estabelecidos }\end{array}$ & $\begin{array}{l}\text { Implementar o produto de acordo com o } \\
\text { design estabelecido } \\
\text { projeto e para o usuário final }\end{array}$ \\
\hline
\end{tabular}

CASTRO, V. S.; OLIVEIRA, S. R. B.; VASCONCELOS, A. M. L.

Um Framework de Práticas Ágeis para Apoio à Implementação do Processo de Projeto e Construção do Produto do MR-MPS-SW

iSys - Revista Brasileira de Sistemas de Informação, Rio de Janeiro, vol. 8, No. 2, p. 78-97 2015 


\begin{tabular}{|l|l|}
\hline \multicolumn{1}{|c|}{ Resultado Esperado } & \multicolumn{1}{|c|}{ Objetivo } \\
\hline $\begin{array}{l}\text { PCP8. A documentação é mantida de acordo com os } \\
\text { critérios definidos }\end{array}$ & $\begin{array}{l}\text { Manter a documentação consistente e } \\
\text { organizada do projeto. }\end{array}$ \\
\hline
\end{tabular}

\section{O Framework de Práticas Ágeis}

A SOFTEX e o SEI - Software Engineering Institute apresentam um conjunto de resultados esperados/práticas específicas para realizar o processo de Projeto e Construção do Produto e área de processo de Solução Técnica, além disso fornecem guias com informações sobre formas de atingir esses resultados esperados, ou seja, guias de implementação.

Essas possibilidades de implementação são descritas de maneira resumida e, na grande maioria das vezes, não apresentam claramente a forma como realizar a implementação. Em SOFTEX (2013), por exemplo é descrito o trecho: "Um processo de seleção de alternativas possui, basicamente, as seguintes atividades: definição dos objetivos de seleção; estabelecimento dos critérios de seleção; desenvolvimento das soluções a serem avaliadas; avaliações das soluções com base nos critérios préestabelecidos e seleção da solução mais adequada.”. Entretanto, não há indicativo de quais objetivos podem ser estabelecidos, como por exemplo, o custo, plataforma de operação. Para organizações que se deparam com esse trecho pela primeira vez pode parecer obscuro o entendimento. Nesse cenário surge a figura da consultoria em processo para detalhar o "como fazer", e isso implica em custos para a organização.

Assim, o desenvolvimento do framework, um conjunto de conceitos usado para resolver um problema de um domínio específico, tem o objetivo de fornecer práticas e formas de execução para apoiar a implementação dos resultados esperados do PCP. As práticas contidas no framework estão definidas nas metodologias ágeis: Crystal, eXtreme Programming, FDD e TDD.

Na subseção 4.1, é apresentada a metodologia de desenvolvimento do framework, bem como a sua estrutura. Já na subseção 4.2, são apresentados dois exemplos de práticas descritas no framework. Devido a limitação de tamanho do artigo, foi disponibilizado na íntegra o framework no endereço etrônico https://drive.google.com/file/d/OBxpLQ8tdmd1TeFVWT201Y1Vjc2c/view?usp=sharing.

\subsection{Metodologia}

A metodologia para o desenvolvimento do framework apresentou as seguintes atividades, como pode ser vista na Figura 1: Revisão da Literatura; Estudo do estado da arte do PCP e das metodologias ágeis; Mapeamento das práticas das metodologias ágeis com os resultados esperados do PCP; Revisão por pares do mapeamento; Desenvolvimento do Framework; e Revisão por pares do framework.

Uma revisão da literatura foi realizada nas principais bases de conhecimento do cenário nacional e internacional. Essa atividade justifica-se pela busca na literatura por referências que possam indicar como o assunto está sendo tratado pela comunidade científica e da indústria de software. Esta etapa forneceu, ainda, subsídios para identificar gaps a serem exploradas sobre o tema da pesquisa.

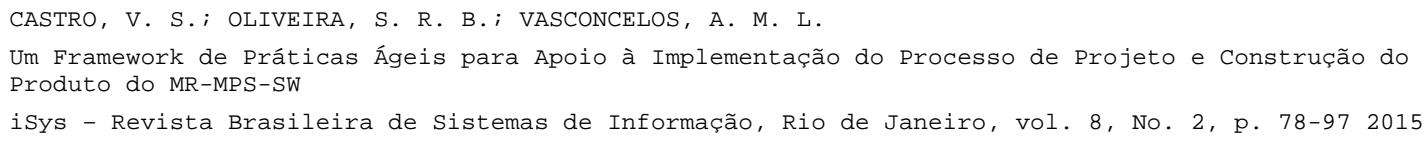


O protocolo de revisão da literatura foi definido em um conjunto de fases visando a melhor estruturação da pesquisa, são elas: Definir os objetivos, a questão de pesquisa, os critérios de seleção e as fontes de dados; Compor a string de busca; Definir os critérios para seleção dos artigos; e Compilar os dados. Um melhor detalhamento da execução e dos resultados obtidos em cada uma destas fases pode ser consultado em [Castro et al., 2015].

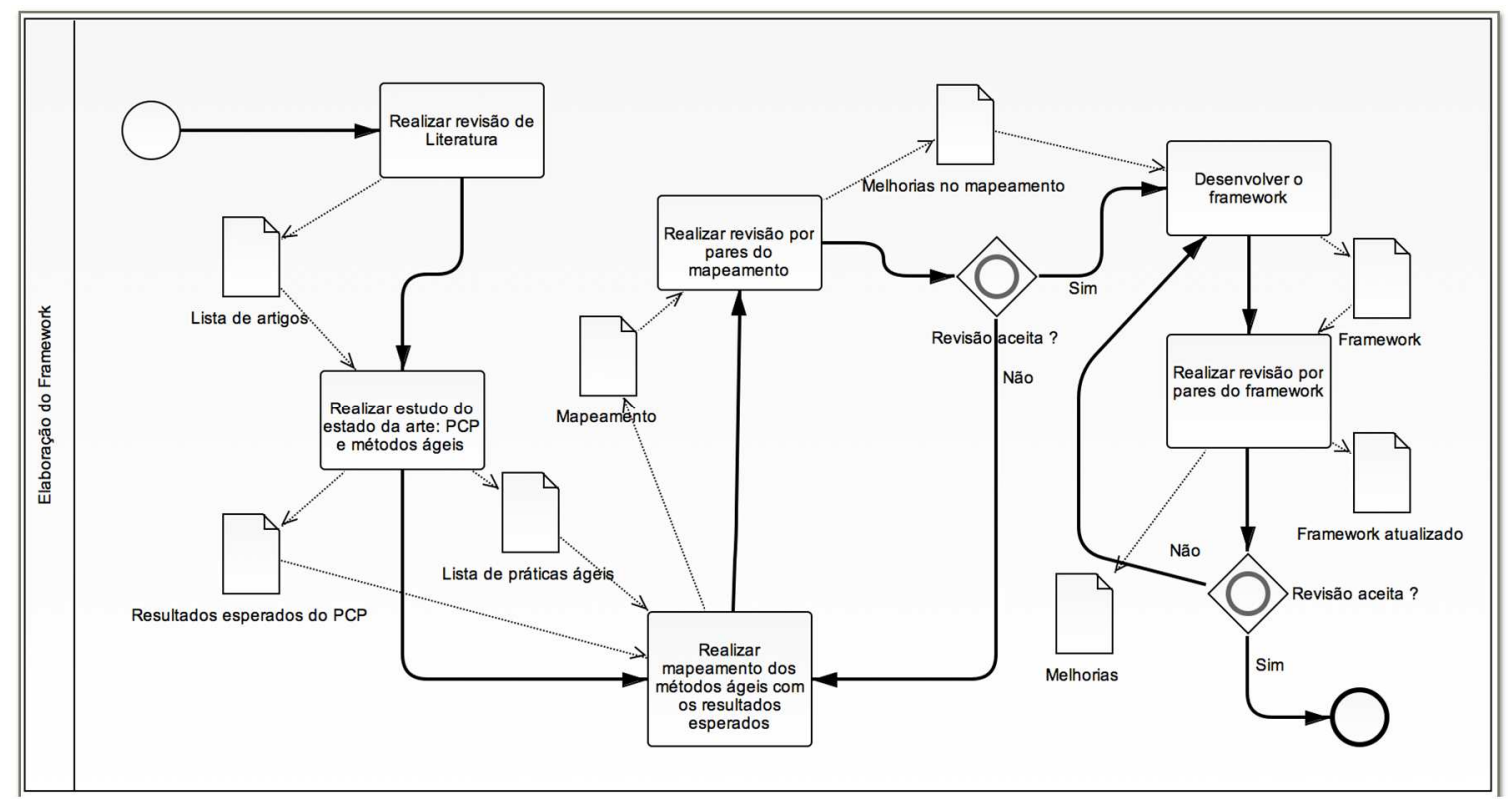

Figura 1. Metodologia de Desenvolvimento do Framework

Esta revisão teve o propósito de alcançar os seguintes objetivos:

- Identificar artigos e trabalhos científicos relacionados ao tema em questão;

- Possibilitar a análise das abordagens e metodologias utilizadas;

- Apresentar um conjunto extenso de práticas utilizadas pela indústria e academia no que diz respeito à área de processo de solução técnica e ao processo de projeto e construção do produto.

Para nortear a revisão da literatura e com intuito de investigar as propostas a questão de pesquisa foi estabelecida: "Quais as metodologias ou práticas ágeis são utilizadas para a implementação do processo solução técnica e projeto e construção do produto ?".

Além dessa questão principal pode-se identificar uma questão secundária: "Dentre as metodologias ágeis encontradas, quais contemplam a implementação de modelos de qualidade ?".

Outra etapa desse protocolo de revisão é a escolha dos critérios para a seleção das fontes de dados. Para a escolha, foram estabelecidos os seguintes critérios:

- Disponibilidade de consulta via web;

- Disponibilidade de artigos em inglês ou português;

CASTRO, V. S.; OLIVEIRA, S. R. B.; VASCONCELOS, A. M. L.

Um Framework de Práticas Ágeis para Apoio à Implementação do Processo de Projeto e Construção do Produto do MR-MPS-SW

iSys - Revista Brasileira de Sistemas de Informação, Rio de Janeiro, vol. 8, No. 2, p. 78-97 2015 
- Disponibilidade na íntegra dos artigos por meio de buscas na internet ou pelo domínio da Universidade Federal do Pará.

As fontes selecionadas foram: IEEE, ACM, EL COMPEDEX e SCOPUS. Além das bases internacionais, foram utilizados os anais do Simpósio Brasileiro de Qualidade de Software (SBQS) e o Workshop Anual do MPS (WAMPS), no período de 2008 a 2013, para contemplar os trabalhos relacionados ao modelo MPS, haja vista que, por exemplo, no WAMPS, os trabalhos contidos nos anais são na totalidade relacionados ao modelo MPS. Além disso, muitos dos trabalhos contidos nos anais do SBQS e do WAMPS não estão disponíveis nas bases internacionais pesquisadas, e sendo o MPS.BR um programa desenvolvido para atender principalmente as organizações no Brasil, o volume de trabalhos em português é mais significativo.

Essa etapa da pesquisa é justificada para ampliar o conhecimento do pesquisador no que tange o assunto da pesquisa e observar quantitativamente e qualitativamente a produção pela comunidade científica. $O$ principal benefício dessa etapa é a identificação dos principais eixos de discussão e possíveis gaps do estudo.

Como resultado da revisão da literatura, um estudo do estado da arte sobre as metodologias ágeis e do processo de PCP foi realizado. Dentre as metodologias encontradas, foram identificadas aquelas que possuem em suas práticas atividades relacionadas à implementação do processo de PCP. A partir das metodologias selecionadas, foi realizado um estudo aprofundado de cada uma das metodologias tendo como base as bibliografias e os artigos selecionados na etapa de revisão.

Além de se estudar as metodologias ágeis, também foi necessário realizar um estudo do estado da arte sobre o processo de PCP. Para a realização desse estudo foram utilizadas as referências da SOFTEX sobre o processo e dos guias definidos pelo SEI, além dos artigos selecionados na etapa de revisão. Esse estudo é justificado para a identificação de quais resultados são esperados por cada uma dessas referências, fornecendo subsídios para a realização do mapeamento das práticas ágeis com o PCP.

Após o estudo sobre as metodologias ágeis e do processo de PCP, foram realizados uma análise e um mapeamento entre as práticas que constam nas metodologias ágeis com os resultados esperados deste processo, no sentido de avaliar se a prática ágil ou um conjunto de práticas contemplam a execução do resultado esperado. Nessa etapa o principal benefício é a identificação de quais práticas ágeis poderão ser utilizadas no framework, e, além disso, quais associações entre as práticas poderão ser realizadas para o alcance dos resultados previstos no PCP.

Para avaliar o mapeamento foi realizada a revisão por pares com um avaliador líder inicial do MPS.BR, sendo possível obter a avaliação necessária por um especialista. A revisão por pares objetiva: avaliar os critérios utilizados para a comparação dos modelos; verificar a aderência entre os elementos presentes nas estruturas dos modelos, quanto a sua correspondência e interpretação dos elementos; e analisar se as considerações feitas esclarecem suas atribuições [Pavan, 2007]. A principal justificativa para a execução dessa etapa é a avaliação do mapeamento realizado e assim fornecer insumos para o desenvolvimento da próxima atividade, o desenvolvimento do framework.

O desenvolvimento do framework foi realizado tendo como base o resultado da revisão por pares e as demais atividades anteriores. Essa atividade teve como principais

CASTRO, V. S.; OLIVEIRA, S. R. B.; VASCONCELOS, A. M. L.

Um Framework de Práticas Ágeis para Apoio à Implementação do Processo de Projeto e Construção do Produto do MR-MPS-SW

iSys - Revista Brasileira de Sistemas de Informação, Rio de Janeiro, vol. 8, No. 2, p. $78-97$ 2015 
elementos: a estruturação de um formato de apresentação do framework; orientação para cada resultado esperado do processo de PCP da implementação por meio das práticas selecionadas das metodologias ágeis contempladas neste trabalho; e, por fim, a elaboração de uma implementação híbrida para o resultado esperado, cujo objetivo é contemplar na totalidade o resultado esperado a partir da junção de duas ou mais práticas das metodologias ágeis.

A Figura 2 apresenta o formato de apresentação do framework, indicando os itens utilizados para descrever o processo de implementação da prática para o resultado esperado.

A Tabela 2 apresenta para cada resultado esperado do PCP as práticas ágeis que estão inseridas no framework. Para a composição do framework foram utilizadas 17 práticas ágeis, sendo que diversas práticas estão aplicadas a mais de um resultado esperado. Como exemplo dessa aplicação, a prática Construir por Feature, que orienta a implementação dos resultados esperados PCP6, PCP7 e PCP8.

Após o desenvolvimento do framework, foi realizada a avaliação por meio da revisão por pares, que será melhor detalhada na Seção 5. 


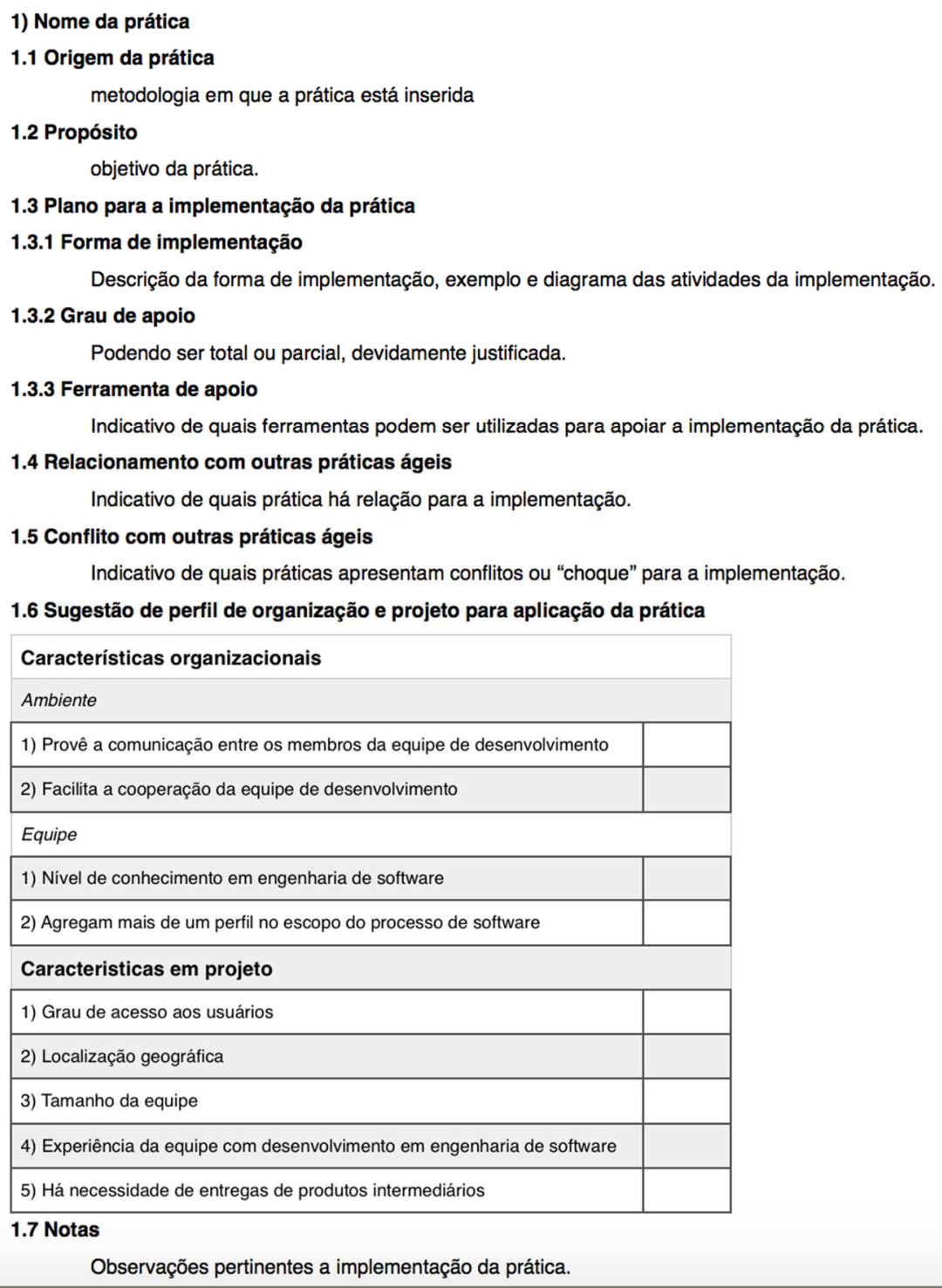

Figura 2. Estrutura do Frawework

\section{Tabela 2. Práticas Ágeis contidas no Framework por Resultado Esperado do Processo de PCP}

\begin{tabular}{|l|l|}
\hline $\begin{array}{l}\text { PCP } \\
1\end{array}$ & $\begin{array}{l}\text { Exploração } 360^{\circ} \text { (Crystal), Rearquitetura incremental (Crystal) e Projeto } \\
\text { Simples (XP) }\end{array}$ \\
\hline $\begin{array}{l}\text { PCP } \\
2\end{array}$ & Esqueleto ambulante (Crystal) e Detalhar por feature (FDD), \\
\hline $\begin{array}{l}\text { PCP } \\
3\end{array}$ & $\begin{array}{l}\text { Padrões de codificação (XP), Projeto Simples (XP), Rearquitetura incremental } \\
\text { (Crystal) e Detalhar por feature (FDD) }\end{array}$ \\
\hline
\end{tabular}




\begin{tabular}{|l|l|}
$\begin{array}{l}\text { PCP } \\
4\end{array}$ & Detalhar por feature (FDD) e Esqueleto ambulante (Crystal), \\
\hline $\begin{array}{l}\text { PCP } \\
5\end{array}$ & Modelagem de Objeto conceitual (FDD) e Exploração $360^{\circ}$ (Crystal), \\
\hline $\begin{array}{l}\text { PCP } \\
6\end{array}$ & $\begin{array}{l}\text { Construir por feature (FDD), Programação por Pares (XP), Testes (XP), } \\
\text { Refatoração (XP), Integração contínua (XP), Esqueleto ambulante (Crystal), } \\
\text { Programação lado a lado (Crystal) e Projetar solução (TDD), }\end{array}$ \\
\hline $\begin{array}{l}\text { PCP } \\
7\end{array}$ & $\begin{array}{l}\text { Documentação abrangente (XP), Manual do usuário (Crystal), Construir por } \\
\text { feature (FDD) e Projetar solução (TDD), }\end{array}$ \\
\hline $\begin{array}{l}\text { PCP } \\
8\end{array}$ & $\begin{array}{l}\text { Documentação abrangente (XP), Detalhar por feature (FDD), Construir por } \\
\text { feature (FDD) e Projetar solução (TDD) }\end{array}$ \\
\hline
\end{tabular}

\subsection{Exemplos do Framework}

Para exemplificar o framework de práticas ágeis, seguem abaixo duas práticas ágeis que apoiam a implementação do PCP 1 e do PCP 6. O PCP 1 tem por objetivo identificar possíveis soluções para a construção do produto com base em um processo de seleção de alternativas. Este resultado possui, basicamente, as seguintes atividades: definição dos objetivos de seleção; estabelecimento dos critérios de seleção; desenvolvimento das soluções a serem avaliadas; avaliações das soluções com base nos critérios préestabelecidos; e seleção da solução mais adequada. Já o PCP 6 é voltado para a implementação do produto de acordo com o design estabelecido e sua documentação deve ser desenvolvida. A inspeção na implementação verificará se o componente do produto foi implementado conforme projetado.

Dentre as práticas ágeis selecionadas para compor o framework tem-se: a Exploração $360^{\circ}$ do Crystal, apoiando o PCP 1; e a Refatoração da eXtreme Programming, apoiando o PCP 6. Estas duas práticas serão utilizadas para exemplificar o framework nas seções 4.2.1 e 4.2.2.

\subsubsection{Exploração 360}

\section{1) Origem da Prática: Crystal}

2) Propósito: Reunião de planejamento das tecnologias, metodologias e processos a serem utilizados no projeto.

3) Plano para a Implementação da Prática

3.1) Forma de implementação: A implementação da prática dá-se inicialmente pela realização de um Brainstorm para levantar alternativas de solução para o projeto. Nessa sessão de brainstorm, líderes de projetos e analistas de sistemas devem trazer inúmeras propostas para a solução do projeto. Aspectos relacionados a padrões de projeto e arquitetura devem fazer parte das discussões. Experiências em projetos correlatos podem apresentar indícios para o reuso de uma solução. Além disso, opções de tecnologias, linguagem de programação e formas de persistências devem ser listadas. 
Para a avaliação da solução, aspectos positivos e negativos devem ser confrontados a fim de direcionar para a classificação da solução. Requisitos como performance, usabilidade, reuso podem ser utilizados como critérios de avaliação das soluções.

Na seleção das possíveis soluções, o uso da metodologia shaping, técnica utilizada para identificar pontos fortes e fracos da organização, pode ser expandido para a análise de alternativas de soluções para o projeto. As soluções que atendem aos critérios estabelecidos pela equipe do projeto devem ser selecionadas. A Figura 3 apresenta o processo de implementação da prática de Exploração $360^{\circ}$.

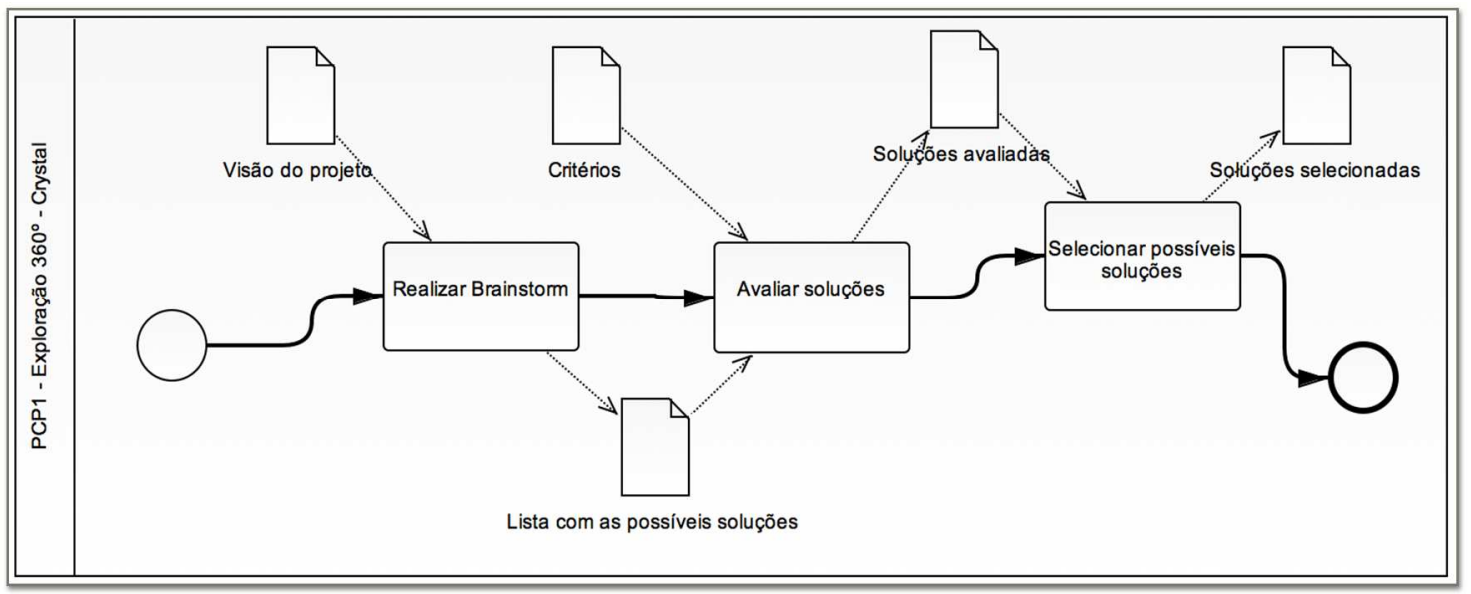

Figura 3. Processo para Execução da Prática Exploração 360‥

3.2) Grau de Apoio: Total, pois por meio da atividade de realização de brainstorm as alternativas de soluções são listadas e, posteriormente, selecionadas pelos critérios estabelecidos.

3.3) Ferramenta de Apoio: uso de quadro branco, post-its e flip-chart são ferramentas que podem auxiliar na execução do brainstorm. Um exemplo do uso seria escrever em cada post-it o identificador da solução e colar no flip-chart, de modo que todos que estão participando da sessão de brainstorm possam visualizar de modo simples as soluções apresentadas como propostas. A mesma técnica pode ser aplicada na avaliação da solução, post-its com aspectos positivos e negativos podem ser agrupados em um quadro branco ou em um flip-chart.

Para equipes que estão trabalhando em locais diferentes, o uso de ferramentas online para video-conferência é fundamental para que ocorra a interação da equipe.

4) Relacionamento com Outras Práticas Ágeis: A implementação dessa prática pode auxiliar a Rearquitetura Incremental, pois fornece uma análise preliminar para a escolha de qual solução pode ser a startup para a evolução e a expansão da solução.

5) Conflito com Outras Práticas Ágeis: Não se aplica.

6) Sugestão de Perfil de Organização e de Projeto para Aplicação da Prática: vide Tabela 3 .

Tabela 3. Sugestão de Perfis Organizacional e de Projeto para a Exploração 360 은

CASTRO, V. S.; OLIVEIRA, S. R. B.; VASCONCELOS, A. M. L.

Um Framework de Práticas Ágeis para Apoio à Implementação do Processo de Projeto e Construção do Produto do MR-MPS-SW

isys - Revista Brasileira de Sistemas de Informação, Rio de Janeiro, vol. 8, No. 2, p. 78-97 2015 


\begin{tabular}{|l|c|}
\hline \multicolumn{2}{|l|}{ Características organizacionais } \\
\hline Ambiente \\
\hline 1) Provê a comunicação entre os membros da equipe de desenvolvimento & Sim \\
\hline 2) Facilita a cooperação da equipe de desenvolvimento & \multicolumn{2}{|l|}{ Sim } \\
\hline Equipe & Alto ou Médio \\
\hline 1) Nível de conhecimento em engenharia de software & \\
\hline 2) Agregam mais de um perfil no escopo do processo de software & Sim \\
\hline Caracteristicas em projeto & Baixo \\
\hline 1) Grau de acesso aos usuários & Qualquer \\
\hline 2) Localização geográfica & Pequena \\
\hline 3) Tamanho da equipe & Alto ou Médio \\
\hline 4) Experiência da equipe com desenvolvimento em engenharia de software \\
\hline 5) Há necessidade de entregas de produtos intermediários & \\
\hline
\end{tabular}

7) Notas: A visão do projeto pode ser apresentada nas formas definidas pela organização, podendo ser: Product Data Sheet, Elevator Statement, Documento de Visão, Lista de requisitos.

Os critérios para a avaliação das soluções deve ter como base as definições do projeto e da equipe.

\subsubsection{Refatoração}

1) Origem da Prática: eXtreme Programming (XP)

2) Propósito: Otimizar o código interno do componente do produto sem a alteração do seu comportamento externo.

3) Plano para a Implementação da Prática

3.1) Forma de implementação: Para iniciar a prática da refatoração, é necessário que haja um componente do produto em desenvolvimento ou concluído. Além disso, um conjunto de boas práticas de programação, padrões de projetos e estilos de programação definidos pela equipe dão a base para a identificação e o projeto da melhoria. Trechos de código que apresentam incompatibilidades, devidamente justificados pela equipe de desenvolvimento, estão sujeitos à prática de refatoração. 
Após a identificação e o projeto da melhoria, a atividade de implementação é iniciada. Os desenvolvedores ajustam o código fonte da aplicação, buscando atingir a melhoria proposta. Essa atividade deve ser documentada, mesmo que em código fonte, para preservar a melhoria. A Figura 4 apresenta o processo de implementação da prática de Refatoração.

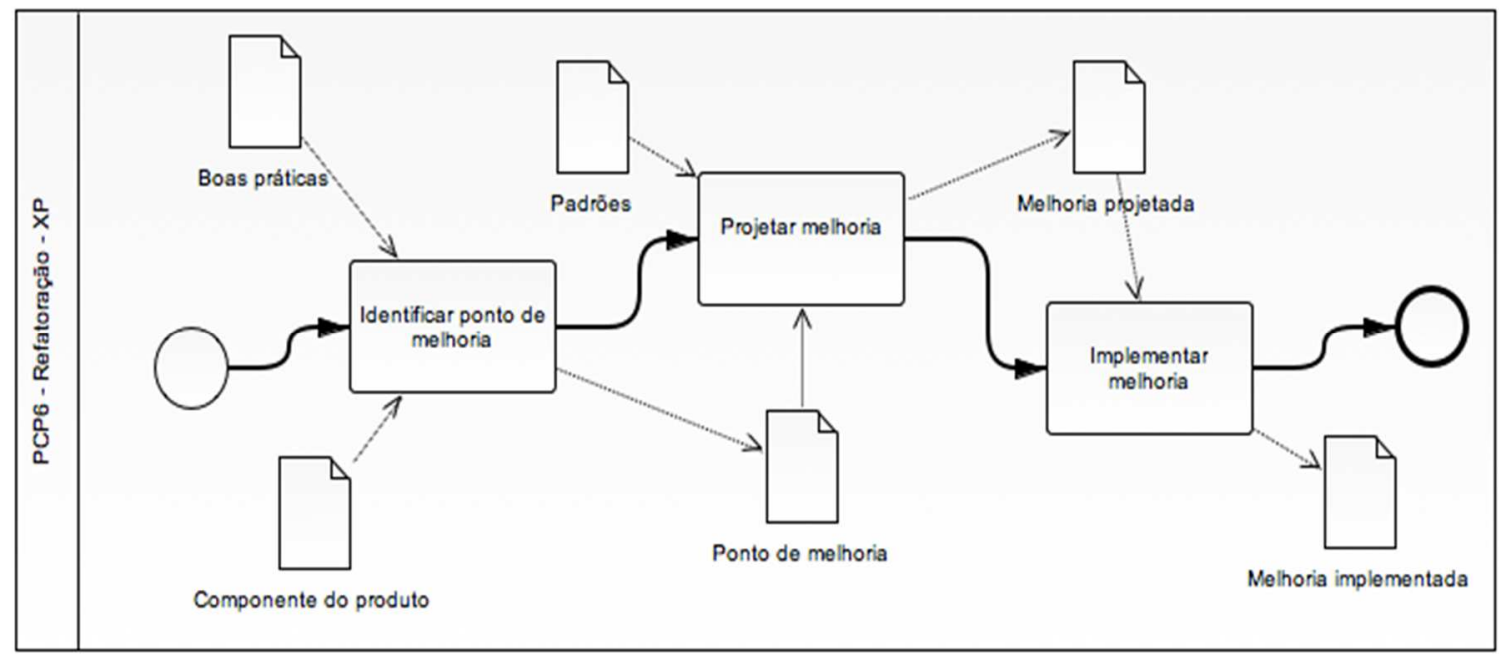

Figura 4. Processo de Execução da Prática de Refatoração

3.2) Grau de Apoio: Parcial, pois, apesar das atividades de implementação do código fonte serem realizadas, não se tem evidência que o processo de verificação é realizado, por conta do objetivo principal da prática que é a permanência do comportamento externo do código, mesmo realizando melhorias/modificações internas do código.

3.3) Ferramenta de Apoio: Ferramentas que identificam estilos de código automaticamente podem ser utilizadas na etapa de identificação do ponto de melhoria.

Para o projeto da melhoria, diagramas UML - Unified Modeling Language (classe, sequência e atividades) podem ser utilizados.

4) Relacionamento com Outras Práticas Ágeis: A Programação por Pares pode ser aplicada em todas as atividades, pois a visão de dois desenvolvedores para discutir o projeto e a forma de implementação pode levar a melhor qualidade na execução da prática.

A associação com a prática de Testes pode ser executada para garantir que o comportamento externo do requisito não será alterado, sendo isso de forma automatizada.

5) Conflito com Outras Práticas Ágeis: Não se aplica.

6) Sugestão de Perfil de Organização e de Projeto para Aplicação da Prática: vide Tabela 4.

\section{Tabela 4. Sugestão de Perfis Organizacional e de Projeto para a Refatoração}

\section{Características organizacionais}

CASTRO, V. S.; OLIVEIRA, S. R. B.; VASCONCELOS, A. M. L.

Um Framework de Práticas Ágeis para Apoio à Implementação do Processo de Projeto e Construção do Produto do MR-MPS-SW

iSys - Revista Brasileira de Sistemas de Informação, Rio de Janeiro, vol. 8, No. 2, p. 78-97 2015 


\begin{tabular}{|l|c|}
\hline Ambiente \\
\hline 1) Provê a comunicação entre os membros da equipe de desenvolvimento & Sim \\
\hline 2) Facilita a cooperação da equipe de desenvolvimento & Sim \\
\hline Equipe & Alto \\
\hline 1) Nível de conhecimento em engenharia de software & Sim \\
\hline 2) Agregam mais de um perfil no escopo do processo de software & \multicolumn{2}{|c|}{} \\
\hline Caracteristicas em projeto & Baixo \\
\hline 1) Grau de acesso aos usuários & Qualquer \\
\hline 2) Localização geográfica & $\begin{array}{l}\text { Média ou } \\
\text { pequena }\end{array}$ \\
\hline 3) Tamanho da equipe & Alto \\
\hline $\begin{array}{l}\text { 4) Experiência da equipe com desenvolvimento em engenharia de } \\
\text { software }\end{array}$ & Sim \\
\hline 5) Há necessidade de entregas de produtos intermediários
\end{tabular}

7) Notas: A prática da Refatoração deve ser priorizada entre os membros da equipe, pois a aplicação de inúmeras refatorações em sequência pode ocasionar um trabalho que "não tem fim", haja vista que se pode implementar um requisito de diversas maneiras.

\section{Revisão por Pares}

A revisão por pares foi realizada para avaliar o framework, tanto a estrutura quanto o conteúdo descritivo. Para realizar essa revisão foi escolhido um especialista com experiência de mais de 5 anos em Engenharia de Software, com elevado conhecimento sobre o processo de Projeto e Construção do Produto do MR-MPS-SW, tendo participado em 3 organizações da implementação do processo PCP.

Além disso, possui certificação em implementação e avaliação nos modelos CMMI-DEV e MR-MPS-SW, sendo considerados o SCAMPI CMMI for Development

Lead Appraiser e o Avaliador Líder no MR-MPS-SW. Este especialista já realizou avaliações em modelos de qualidade em mais de 10 organizações de software e tem experiência em avaliação de mais de 5 anos. No contexto das metodologias ágeis em estudo nesse artigo, o avaliador possui conhecimento médio e experiência em mais de 5 anos. itens:

O processo de avaliação por pares teve como critérios de avaliação os seguintes 
- TA (Técnico Alto), indicando que foi encontrado um problema em um item que, se não for alterado, comprometerá as considerações;

- TB (Técnico Baixo), indicando que foi encontrado um problema em um item que seria conveniente alterar;

- E (Editorial), indicando que foi encontrado um erro de português ou que o texto pode ser melhorado;

- Q (Questionamento), indicando que houve dúvidas quanto ao conteúdo das considerações;

- G (Geral), indicando que o comentário é geral em relação às considerações;

- $\quad \mathbf{N}$ (Notas sobre as práticas), indicando que o comentário pode ser adicionado na forma de Notas para a prática ágil.

Foi fornecido ao especialista uma planilha eletrônica para ser utilizada como suporte para anotações acerca dos itens, contendo a categoria (critério de avaliação), o item em que a categoria foi aplicada, os comentários com justificativa sobre o item avaliado e a sugestão para correção/melhoria.

O processo de avaliação por pares foi realizado no mês de Janeiro de 2015, sendo em três momentos: no primeiro foi enviado para o especialista o framework e a planilha eletrônica com os critérios de avaliação e um formulário de avaliação geral; no segundo, devido ao volume de informação contido no framework, foram realizadas reuniões remotas para a apresentação do material; e, por fim, o terceiro momento consistiu na apresentação por parte do especialista da planilha de avaliação preenchida e o indicativo de sugestões/melhorias. Na Tabela 5 e na Figura 5 há o quantitativo indicado pelo especialista por critério de avaliação.

Tabela 5. Dados da Revisão por Pares

\begin{tabular}{|l|c|}
\hline Critérios & Quantidade \\
\hline TA - Técnico Alto & 2 \\
\hline TB - Técnico Baixo & 18 \\
\hline E - Editorial & 14 \\
\hline Q - Questionamento & 0 \\
\hline G - Geral & 3 \\
N - Notas sobre as práticas & 0 \\
\hline
\end{tabular}

CASTRO, V. S.; OLIVEIRA, S. R. B.; VASCONCELOS, A. M. L.

Um Framework de Práticas Ágeis para Apoio à Implementação do Processo de Projeto e Construção do Produto do MR-MPS-SW 


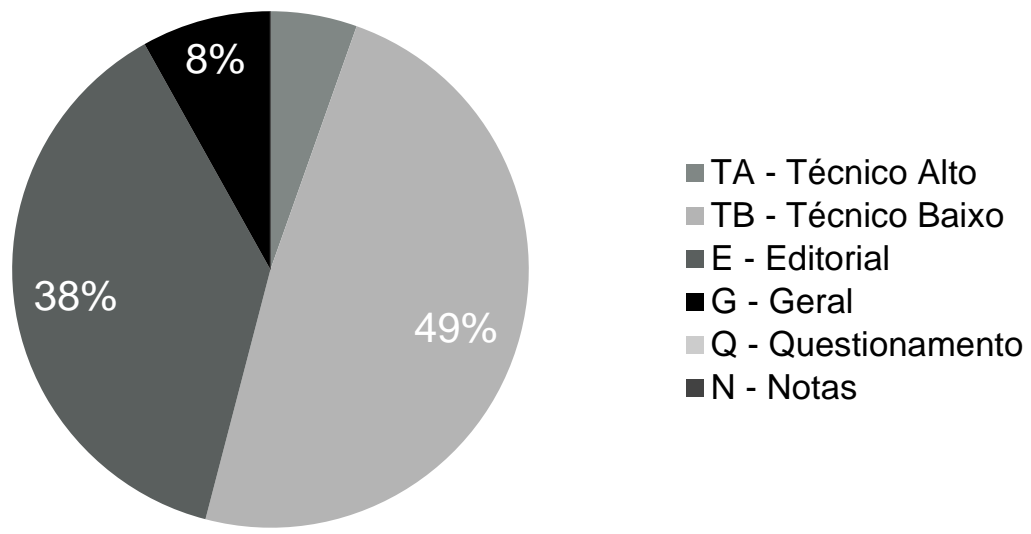

Figura 5. Gráfico do Resultado da Revisão por Pares

Após o recebimento da planilha de avaliação, todos os problemas identificados foram corrigidos, de acordo com a sugestão descrita pelo especialista. Finalizadas as correções, o framework foi encaminhado novamente para o especialista para aprovação. Todas as correções realizadas foram aprovadas pelo especialista.

Diante de 2 itens categorizados como TA - Técnico Alto, as seguintes correções foram realizadas, pois essa categoria denota que há um problema e se não for alterado, comprometerá as considerações, são eles:

- Comentário: "Justificar o item grau de apoio para todas as práticas". Esse comentário foi considerado pertinente e para todas as práticas do framework foi justificado o grau de apoio, para ambos os casos (Total ou Parcial).

- Item: PCP3 - Prática padrões de codificação, comentário: "No item das características de projeto: Há necessidade de entregas de produtos intermediários? O valor deve ser SIM, pois a prática gera produtos intermediários". Nesse item a justificativa foi acatada e para o item a mudança de NÃO para SIM foi realizada.

Na Figura 6 foram identificados para cada resultado esperado os itens de avaliação TA - Técnico Alto, TB - Técnico Baixo e E - Editorial. Nota-se que o resultado esperado PCP 6 possui o maior quantitativo de itens de avaliação: TB, com 6 itens; e E, com 7 itens. Já o resultado esperado PCP 2 não contabilizou nenhum item de avaliação. A categoria G - Geral não foi incluída como indicador para a Figura 6, em razão de sua característica, aplicada a todos os resultados esperados. Alguns itens categorizados como TA, TB e E não estão indicados no gráfico pois foram aplicados a todo o framework. 


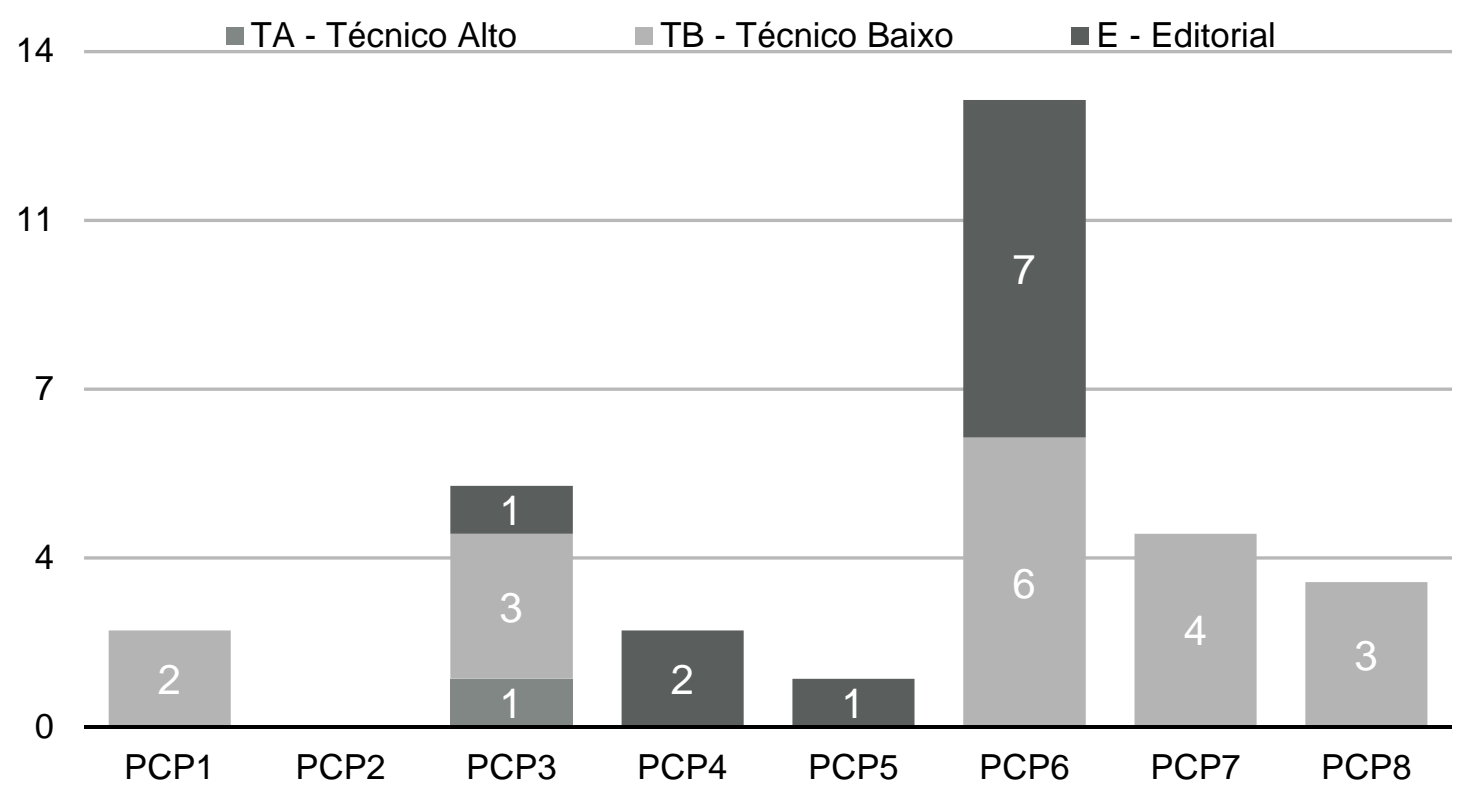

Figura 6. Itens de avaliação por resultado esperado

Sobre a formulário de avaliação geral, o especialista classificou a proposta como "Atende Totalmente" e considerou a estrutura "Completa". Citou que a metologia ágil Lean poderia ter sido contemplado no framework. Além disso considerou a proposta um referencial de apoio para a implementação do processo de PCP com práticas ágeis e indicou que "Adoto atualmente na implementação do CMMI-DEV e MR-MPS-SW em uma organização de software".

\section{Conclusões}

Diante do problema discutido sobre o apoio à implementação dos procesos de Engenharia de Software constantes nos modelos de qualidade a partir do uso de práticas presentes me métodos ágeis, a principal contribuição do trabalho é a apresentação de um arcabouço de práticas ágeis, por meio de um framework, que discute como aplicar estas práticas para alcançar o propósito definido para o processo de PCP. Ao longo do trabalho pôde-se perceber a existência de inúmeros trabalhos que não apresentam a forma de implementação da prática e sua associação aos resultados esperados do processo de PCP. No framework de práticas ágeis essa associação é realizada.

Outra contribuição do trabalho está relacionada ao fornecimento de um referencial teórico para organizações que estão implementando modelos de qualidade com métodos ágeis, especificamente o processo de PCP constante no MR-MPS-SW. O detalhamento da forma de implementação de cada prática ágil apresenta para a organização um possível apoio para o alcance das metas definidas ao processo.

Como limitações do trabalho pode-se destacar: (i) a não realização do mapeamento levando em consideração os processos que possuem relacionamentos com o processo de PCP, tais como o Desenvolvimento de Requisitos, relacionado à concepção do produto, e os processos de Verificação e Validação, relacionados com a aplicação das técnicas de Teste e Revisão por Pares; (ii) apesar da realização da revisão por pares do framework como forma de avaliação, um processo de experimentação não foi realizado

CASTRO, V. S.; OLIVEIRA, S. R. B.; VASCONCELOS, A. M. L.

Um Framework de Práticas Ágeis para Apoio à Implementação do Processo de Projeto e Construção do Produto do MR-MPS-SW

isys - Revista Brasileira de Sistemas de Informação, Rio de Janeiro, vol. 8, No. 2, p. 78-97 2015 
para validá-lo; e (iii) o número de metodologias do framework, pois no processo de revisão por pares houve o indicativo do uso do método Lean para atendimento dos resultados esperados do processo de PCP.

Como trabalhos futuros pretende-se realizar a aplicação do framework no contexto das organizações que fazem uso de práticas ágeis para apoiar a implementação de modelos de qualidade. Além disso, pretende-se realizar o estudo da metodologia Lean no contex to do atendimento aos resultados esperados do MR-MPS-SW. Outro trabalho futuro refere-se à expansão do framework para os demais resultados esperados constantes em outros processos do modelo de qualidade.

\section{Agradecimentos}

Esse trabalho faz parte do projeto SPIDER - Software Process Improvement: DEvelopment and Research, institucionalizado na UFPA - Universidade Federal do Pará (www.spider.ufpa.br).

\section{Referências}

Beck, K. (2003) “Test Driven Development: By Example”. Pearson Education.

Beck, K. (2004) "Programação extrema explicada: acolha as mudanças". Bookman.

Castro, V. S., Oliveira, S. R. B., Vasconcelos, A. M. L. (2015) "Uma Análise da Literatura Especializada sobre a Aplicação de Métodos Ágeis no Contexto da Área de Processo de Solução Técnica constante em Modelos de Qualidade para Processo de Software". Revista de Sistemas de Informação da FSMA, n 15, pp. 52-59.

Catunda, E., Nascimento, C., Cerdeiral, C., Santos, G., Nunes, E., Schots, N., Chaves, L., Schots, M., Rocha, A. R. (2011) "Implementação do Nível F do MR-MPS com Práticas Ágeis do Scrum em uma Fábrica de Software”. Anais do SBQS'2011.

Cockburn, A. (2002) "Crystal Clear - A Human-Powered Methodology For Small Teams, including The Seven Properties of Effective Software Projects".

Hansen, M., Baggesen, H. (2009) "From CMMI and isolation to Scrum, Agile, Lean and collaboration".

the International Organization for Standardization and the International Electrotechnical Comission (2004) "ISO/IEC 15504-1: Information Technology - Process Assessment - Part 2: Performing an Assessment". Geneve.

Lukasiewicz, K., Miler, J (2011) "Improving agility and discipline of software development with the Scrum and CMMI".

Pavan, C., STUMPF, I. R. C. (2007) "Revistas Brasileiras de Ciência da Computação: procedimentos de avaliação pelos pares". VIII ENANCIB - Encontro Nacional de Pesquisa em Ciência da Computação.

Pressman, R. (2011) “Engenharia de Software”. Sétima Edição, Bookman.

Poppendieck, M., Poppendieck, T (2011) “Implementando o desenvolvimento LEAN: do conceito ao dinheiro". Bookman.

Retamal, A. (2008) "Feature-Driven Development".

CASTRO, V. S.; OLIVEIRA, S. R. B.; VASCONCELOS, A. M. L.

Um Framework de Práticas Ágeis para Apoio à Implementação do Processo de Projeto e Construção do Produto do MR-MPS-SW

isys - Revista Brasileira de Sistemas de Informação, Rio de Janeiro, vol. 8, No. 2, p. 78-97 2015 
Sbrocco, J., Macedo, P. (2012) "Metodologias Ágeis - Engenharia de Software sob medida". Ética.

SEI - Software Engineering Institute (2010) "CMMI for Developement - version 1.3". Disponível em: http://www.sei.cmu.edu/reports/10tr033.pdf. Último acesso: Abril/2015.

Silva, T., Magela, R., Santos, G., Schots, N., Chaves, L., Rocha, A. R. (2011) "Implantação do Nível F do MR-MPS Combinando Características do Processo Unificado com Práticas SCRUM”. Anais do WAMPS'2011.

SOFTEX. (2013) "Guia de Implementação - Parte 4: Fundamentação para Implementação do Nível D do MR-MPS".

SOFTEX (2015), "Resultado das Avaliações MPS-SW publicadas em 2015". Disponível em: $\quad h t t p: / / w w w . s o f t e x . b r / w p-c o n t e n t / u p l o a d s / 2013 / 07 / 2 A v a l i a c o e s-M P S S W$ Publicadas_26.jun_.2015_649.pdf. Último acesso: Julho/2015.

Sommerville, I. (2007) "Engenharia de Software”. 8 edição, Pearson Addison-Wesley. 